\title{
CONGRÈS D'HISTOIRE \\ DES SCIENCES \\ ET DES TECHNIQUES \\ LILLE, 24-26 MAI 2001
}

La Société française d'histoire des sciences et des techniques, ouverte à tous les membres de la communauté des historiens des sciences et des techniques, a voulu organiser ce congrès afin de faire le point sur l'ensemble des activités et des travaux menés ces dernières années, qu'il s'agisse d'enseignement, de recherche ou de publication. Nous avons estimé que cette réunion sert les intérêts de la communauté des historiens des sciences et des techniques, contribuant à sa connaissance mutuelle, à l'appréciation ou à la critique de ses propres activités, à l'intensification de ses rapports internes.

Le congrès de Lille devrait être l'occasion d'avancer des propositions pour contribuer à l'amélioration de la situation de l'histoire des sciences et des techniques en France.

Enfin, il participera à la valorisation de la contribution française en ces domaines face aux communautés et sociétés étrangères correspondantes.

Ce pourrait être la première d'une suite de telles réunions, régulièrement organisées.

Bernard Joly et Vincent JuLliEN

Directeurs du Congrès

Responsables de sessions

Session 1 : Dominique Lecourt et Véronique Le Ru, Enseignement de l'histoire des sciences et des techniques.

Session 2 : Antoine Picon et Hélène Vérin, Histoire des techniques, des cultures matérielles et des industries.

Session 3 : Pietro Corsi et Claude Debru, Histoire des sciences du vivant et de son environnement.

Session 4: Robert Locqueneux et Michel Blay, Histoire de la physique, de la chimie et des sciences de l'Univers.

Session 5 : Thérèse Charmasson et Christiane Demeulenaere-Douyère, Les matériaux de l'histoire.

Session 6: Evelyne Barbin et Éric Brian, Histoire des mathématiques, de la logique et de l'informatique.

Session 7 : Dominique Pestre et Pascal Brioist, Histoire des acteurs, réseaux, institutions, usages et langage de la science.

\section{Secrétariat du Congrès}

Fatima Abdelouahab

Tél. : 33 (0)3.20.91.80.63

Fax : 33 (0)3.20.41.67.14

Courrier électronique : sfhst@univ-lille3.fr

Revue de synthèse : $4^{\mathrm{e}} \mathrm{S} . \mathrm{n}^{\mathrm{o}} 1$, janv.-mars 2001, p. 253. 